\title{
Tests of hadronic interactions using the Pierre Auger Observatory
}

\author{
Patricia M. Hansen ${ }^{* \dagger}$ \\ Instituto de Física de La Plata (CCT La Plata-CONICET), \\ Departamento de Física, Facultad de Ciencias Exactas y \\ Departamento de Ciencias Básicas, Facultad de Ingeniería, \\ Universidad Nacional de La Plata, \\ C. C. 67 - 1900 La Plata, Argentina \\ E-mail: hansen.patricia@gmail.com
}

\section{The Pierre Auger Collaboration}

Observatorio Pierre Auger, Av. San Martín Norte 304, 5613 Malargüe, Argentina

E-mail: auger_spokesperson@fnal.gov

Full author list: http://www.auger.org/archive/authors_2018_07.html

Ultra-high energy cosmic rays (UHECR) can be used to study hadronic interactions beyond LHC energies. In this contribution, we summarize relevant data from the Pierre Auger Observatory. While the proton-air cross section has been measured at $\sqrt{s}=57 \mathrm{TeV}$ and found to be in good agreement with extrapolations from LHC energies, other observables are significantly different from what is predicted using current models. In particular, the predictions from models of showers based on LHC data are in strong contradiction with the observed number of muons. More muons are detected than predicted with the magnitude of the effect being model-dependent. Another observable from the Pierre Auger Observatory, the distribution of the depths of muon production, is also poorly described. Indeed no current model is capable of describing the full range of data from the Observatory, thus highlighting deficiencies in extrapolations beyond LHC energies. The discrepancy between hadronic models can be examined further by measuring separately the muon and electromagnetic components of the signal recorded by detectors on the ground. The Pierre Auger Observatory is being upgraded by the addition of plastic scintillator detectors above the water-Cherenkov detectors to achieve this. The new observations will enable rigorous testing of hadronic models up to $\sqrt{s} \sim 100 \mathrm{TeV}$ and are crucial to the quest of determining the composition of UHECR.

The 39th International Conference on High Energy Physics (ICHEP2018)

4-11 July, 2018

Seoul, Korea

\footnotetext{
${ }^{*}$ Speaker.

${ }^{\dagger}$ for the Pierre Auger Collaboration
} 


\section{Introduction}

The Data on UHECR recorded at the Pierre Auger Observatory [1][2] can be used to study hadronic interactions beyond the LHC energy. In this contribution, we summarize some of the results of the Pierre Auger Observatory in this branch. We make a comparison between data on UHECR-induced showers collected at the Pierre Auger Observatory and predictions from models of hadronic interactions based on LHC data for the following observables:

(a) The muon production depth [3] [4] [5]. (b) Mean number of muons in highly inclined air showers [6] [7].

There are others results of the Pierre Auger Observatory in this field (that I will not describe here)

(c) The muon content of hybrid events (detected simultaneously with the fluorescence and surface detector) [8] [9]. (d) The time profile of the signals recorded with the water-Cherenkov detectors [10]. (e) The azimuthal asymmetry in the rise time of signals in the Auger surface detector [11].

\section{Muon production depth}

All events recorded at the surface detector (SD) of Auger between January 2004 and December 2016 have been used in the analysis. Using the selection criteria and the ranges of applicability that are explained in [3] [4] [5], the number of UHECR analyzed in this work is 2227. In figure 1 (left) the data have been studied as a function of primary energy. Black squares correspond to data with their statistical uncertainties and the gray band to the systematic uncertainties. For each energy bin, the number of events is indicated. The solid line corresponds to the predicted $\left\langle X_{\max }^{* \mu}\right\rangle$ using QGSJETII-04 [12] and the dotted line to the prediction using EPOS-LHC [13]. For EPOS-LHC the data does not agree with predictions for all reasonable mass in the whole energy range. In the case of QGSJETII-04 this model agrees with iron expectation but there are incompatibilities at higher energies. We can also observe that the prediction of $\left\langle X_{\max }^{* \mu}\right\rangle$ from the two hadronic models are significantly different in absolute value $\left(35 \mathrm{~g} / \mathrm{cm}^{2}\right)$. However, we can note that the muonic elongation rate (the rate of change $\left\langle X_{\max }^{* \mu}\right\rangle$ with primary energy) is predicted to be about $\sim 25 \mathrm{~g} / \mathrm{cm}^{2} /$ decade independently of the primary mass and hadronic model, while on data we found $-16.9 \pm 7.2 \mathrm{~g} / \mathrm{cm}^{2} /$ decade. Finally, further information about the consistency of the hadronic interaction models can be obtained by converting $\left\langle X_{\max }^{* \mu}\right\rangle$ to $\ln A$ (with $A$ the mass number), by using the following formula:

$$
\langle\ln A\rangle=\ln 56 \frac{\left\langle X_{\max }^{* \mu}\right\rangle \mathrm{p}-\left\langle X_{\max }^{* \mu}\right\rangle}{\left\langle X_{\max }^{* \mu}\right\rangle \mathrm{p}-\left\langle X_{\max }^{* \mu}\right\rangle_{\mathrm{Fe}}},
$$

where $\left\langle X_{\max }^{* \mu}\right\rangle_{\mathrm{p}}$ and $\left\langle X_{\max }^{* \mu}\right\rangle_{\mathrm{Fe}}$ are the average values for proton and iron-induced air showers respectively, obtained after the reconstruction. $\left\langle X_{\max }^{* \mu}\right\rangle$ is the average measured value for the data. This comparison can be performed because the linear relation between $\left\langle X_{\max }^{* \mu}\right\rangle$ and $\ln A$ has been checked and verified by means of a set of Monte Carlo simulations for different nuclei. The results are shown in figure 1 (middle and right) together with $\langle\ln A\rangle$ from electromagnetic $X_{\max }$ [3] [5], for each model separately, EPOS-LHC (middle) and QGSJETII-04 (right). The results are not compatible both for QGSJETII-04 $(\approx 3.3 \sigma)$, and for EPOS-LHC $(\approx 6 \sigma)$. 

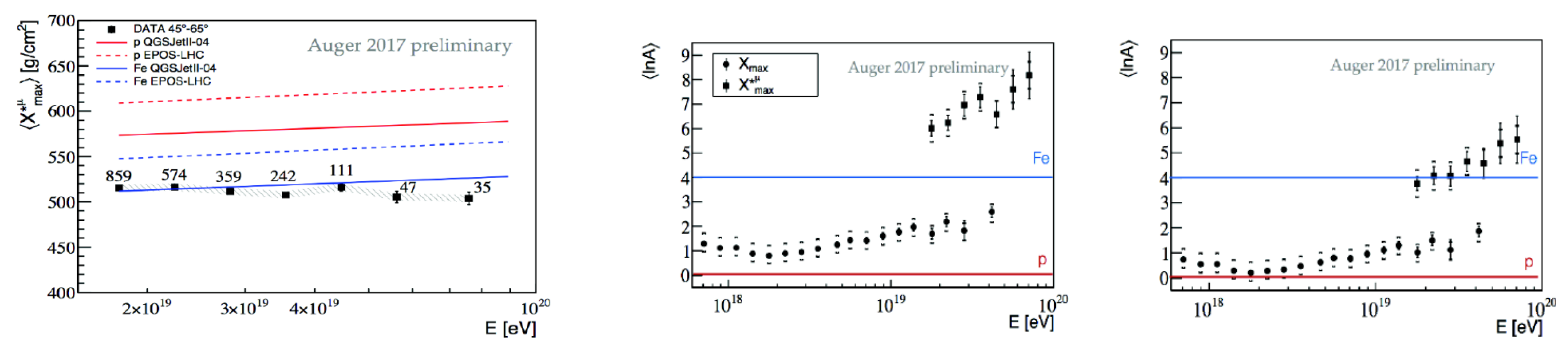

Figure 1: Left: $\left\langle X_{\max }^{* \mu}\right\rangle$ as a function of the primary energy. Data (black squares) are shown with statistical (black line) and systematic uncertainties (gray band) and compared to simulation (see text for the details). Middle - right: The evolution with energy of $\langle\ln A\rangle$ as obtained from $X_{\max }^{* \mu}$ (squares). The results obtained for $X_{\max }$ (dots) are also shown. EPOS-LHC (middle) and QGSJETII-04 (left) are used as reference models. Square brackets correspond to the systematic uncertainties.

\section{Mean number of muons in highly inclined air showers}

To study the muon content $R_{\mu}$ (see [6]) of inclined showers as a function of the cosmic-ray energy $E$ we use $N_{19}$ for energy calibration. This is done using the calorimeter energy from highquality events measured simultaneously with $S D$ and $F D$ (fluorescence detector). In the same way we obtain the correlation of $R_{\mu}$ with $E_{F D}$ to study the relative muon content of measured showers as a function of energy. We expect that the average number of muons, which is proportional to $\left\langle R_{\mu}\right\rangle$ follows the relation:

$$
\left\langle R_{\mu}\right\rangle=\alpha\left(E / 10^{19} \mathrm{eV}\right)^{b}
$$

From figure 2 (left) we found that at energy $10^{19} \mathrm{eV},\left\langle R_{\mu}\right\rangle=1.84$. But using CORSIKA [16] simulation with QGSJETII-03 [14] and FLUKA [15] interaction models at zenith angle $67^{\circ}$ (that correspond to the average zenith angle of the data) and energy $10^{19} \mathrm{eV},\left\langle R_{\mu}\right\rangle=1$. So we conclude that QGSJETII-03 under-predicts the number of muons at $10^{19} \mathrm{eV}$ by a factor 1.8 .
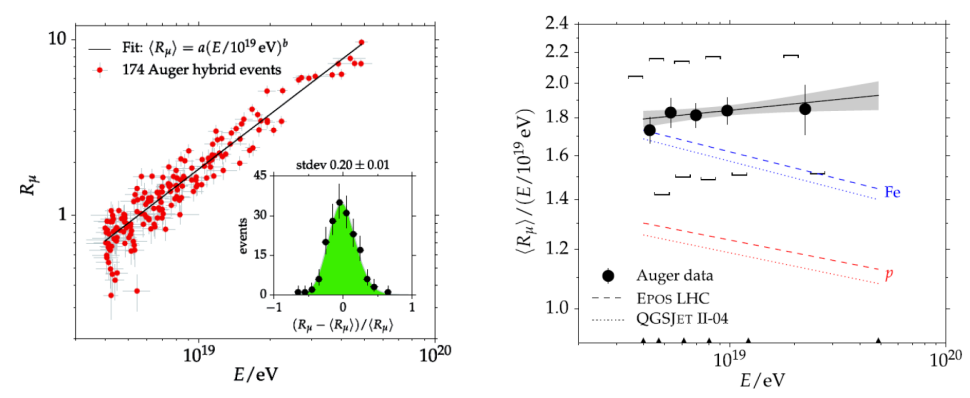

Figure 2: Left: The selected hybrid events above $4 \times 10^{18} \mathrm{eV}$ and a fit of the power law: $\left\langle R_{\mu}\right\rangle=$ $\alpha\left(E / 10^{19} \mathrm{eV}\right)^{b}$. The error bars indicate statistical detection uncertainties only. The inset shows a histogram of the residuals around the fitted curve (black dots) and for comparison the expected residual distribution computed from the fitted probability model that describes the fluctuations. Right: Average muon content $\left\langle R_{\mu}\right\rangle$ per shower energy $E$ as a function of the shower energy $E$. Our data (circles) is shown together with the fit (line). Square brackets indicate the systematic uncertainty of the measurement. The grey band indicates the statistical uncertainty of the fitted line. Shown for comparison are theoretical curves for proton and iron showers simulated at $\theta=67^{\circ}$ (dotted and dashed lines). 
In figure 2 (right) we compare our data with the prediction of an air shower simulation program for primary proton and iron showers at zenith angle $67^{\circ}$ with hadronic model QGSJETII-04 and EPOS-LHC. We display the ratio $R_{\mu} /\left(E / 10^{19} \mathrm{eV}\right)$ because it cancels most of the energy scaling and emphasizes the effect of cosmic-ray mass $A$ on the muon number. For the data, we divide them on energy bins and we compute the ratio. We can see that proton and iron showers are well separated which illustrates the power of $\left\langle R_{\mu}\right\rangle$ as a composition estimator. The main discrepancy between model and the data is the high abundance of muons in the data. The measured muon number is higher than in pure iron showers, suggesting a contribution of heavier elements.

\section{Conclusion}

Auger is indeed contributing to hadronic physics at ultra high energy. We compare different observables obtained by the Pierre Auger Observatory with predictions from models of hadronic interactions based on LHC data. No current model is capable of describing the full range of data from the Pierre Auger Observatory, thus highlighting deficiencies in extrapolation beyond LHC energies. -The muon production depth: EPOS-LHC vs data: inconsistency with predictions for all reasonable primary mass $(\mathrm{p} / \mathrm{Fe})$, in the whole energy range. QGSJETII-04 vs data: consistent with iron expectation but not at the highest energies. The prediction of the two hadronic models are significantly different in absolute value $\left(\sim 35 \mathrm{~g} / \mathrm{cm}^{2}\right)$. The muonic elongation rate is predicted to be about $\sim 25 \mathrm{~g} / \mathrm{cm}^{2} /$ decade, independently of the primary mass and hadronic models, while on data we found $-16.9 \pm 7.2 \mathrm{~g} / \mathrm{cm}^{2} /$ decade. -Mean number of muons in highly inclined events: We observe a muon deficit in simulation of 30 to $80 \%$ at $10^{19} \mathrm{eV}$, depending on the model and primary particle $(\mathrm{p} / \mathrm{Fe})$.

\section{References}

[1] A. Aab el al, Pierre Auger Collaboration, NIMA 798 (2015) 172.

[2] Michael Unger, for the Pierre Auger Collaboration, Proc. 35th ICRC2017, Bexco, Busan, Korea. POS (ICRC2017) 1102 (2017).

[3] A. Aab et al. Pierre Auger Collaboration, Phys. Rev. D 90 (2014) 122005.

[4] Laura Collica, for the Pierre Auger Collaboration, Eur. Phys. J. Plus (2016) 131: 301.

[5] Manuela Mallamaci, for the Pierre Auger Collaboration, Proc. 35th ICRC2017, Bexco, Busan, Korea. POS (ICRC2017) 509 (2017).

[6] A. Aab et al. Pierre Auger Collaboration, Phys. Rev. D 91032003 (2015).

[7] Inés Valiño, for the Pierre Auger Collaboration, Proc. 33th ICRC2013, Rio de Janeiro, Brazil (2013).

[8] A. Aab et al. Pierre Auger Collaboration, Phys. Rev. Lett. 117, 109001 (2016).

[9] G. R. Farrar, for the Pierre Auger Collaboration, Proc. 33th ICRC2013, Rio de Janeiro, Brazil (2013).

[10] A. Aab et al. Pierre Auger Collaboration, Phys. Rev. D 96, 122003 (2017).

[11] A. Aab et al. Pierre Auger Collaboration, Phys. Rev. D 93, 072006 (2016).

[12] S. S. Ostapchenko, Phys. Rev. D 83, 014018 (2011)

[13] T. Pierog, Iu. Karpenko, J.M. Katzy, E. Yatsenko, and K. Werner, Report No. DESY-13-125, 2013

[14] S. S. Ostapchenko, Phys. Rev. D 74, 014026 (2006)

[15] A. Ferrari el al., CERN-2005-10 (2005) INFN/TC-05/11, SLAC-R-773.

[16] D. Heck, G. Schatz, T. Thouw, J. Knapp, and J.N. Capdevielle, Report N N $_{0}$ FZKAA 6019, 1998. 